\title{
Image-guided interstitial brachytherapy boost for nasopharyngeal carcinoma: technical aspects
}

\author{
Naoya Murakami, MD, PhDl, Guanghui Cheng, MD, PhD², Seiichi Yoshimoto, MD, PhD³, Jun Itami, MD, PhD' \\ 'Department of Radiation Oncology, National Cancer Center Hospital, Tokyo, Japan, ${ }^{2}$ Department of Radiation Oncology. China-Japan \\ Union Hospital of Jilin University, Changchun, China, ${ }^{3}$ Department of Head and Neck Surgery, National Cancer Center Hospital, Japan
}

\begin{abstract}
Nasopharyngeal cancer generally responds well to concurrent chemoradiotherapy (CCRT). However, there is a small group of patients who respond poorly to CCRT, and experience local residual tumor or local relapse. Although several attempts have been performed to such a group of patients including re-irradiation with external beam radiation therapy (EBRT) or salvage surgery, clinical results remain unsatisfactory. Intracavitary brachytherapy (ICBT) boost after CCRT with EBRT has been explored, however, its efficacy is limited to those with superficial residual tumors. For those residual tumors thickness with more than $5 \mathrm{~mm}$, interstitial brachytherapy (ISBT) boost would be an appropriate modality of choice.

Here, we describe technical aspects of the high-dose-rate interstitial brachytherapy (HDR-ISBT) boost for nasopharyngeal cancer (NPC) patients who responded poorly to the CCRT with EBRT.
\end{abstract}

J Contemp Brachytherapy 2020; 12, 3: 294-302

DOI: httos://doi.org/10.5114/jcb.2020.96874

Key words: nasopharyngeal carcinoma, interstitial brachytherapy boost, image-guided brachytherapy.

\section{Purpose}

Because of its anatomical complexity, it is generally difficult to safely insert interstitial brachytherapy applicators in the head and neck region compared to other anatomical sites such as prostate, breast, or uterine cervix. Therefore, interstitial brachytherapy (ISBT) is not widely performed in the head and neck region except for some expert institutions. In the National Comprehensive Cancer Network guidelines for head and neck cancers version 1.2020, it is stated that brachytherapy is now being used less often because of improved local control obtained with concurrent chemoradiation, and brachytherapy has a role for lip and oral cavity cancers [1]. Lack of efficient training system availability for head and neck ISBT, and the emergence of more sophisticated delivery of external radiotherapy such as intensity-modulated radiation therapy or volumetric modulated arc therapy, with the effectiveness of concurrent chemoradiotherapy also contributed to diminished usage of head and neck ISBT. There have been reports on the head and neck ISBT such as early-stage tongue cancer $[2,3,4,5,6,7,8]$, oral cavity cancers $[9,10,11,12]$, oropharyngeal cancers $[5,13,14]$, or nasal vestibules [15], and its usefulness on local control and survival has been demonstrated.

The vast majority of nasopharyngeal cancer (NPC) consists of non-keratinizing squamous cell carcinoma (NKSCC) and it is well-known that carcinogenesis of NKSCC is related to Epstein-Barr virus (EBV) infection $[16,17]$. While most NKSCC NPC responds well to ra- diation therapy or chemotherapy, it has been reported that unfortunately, approximately $7.5-14 \%$ of locally advanced NPC patients develop persistent or local relapse after definitive concurrent chemoradiotherapy (CCRT) $[18,19,20]$. Prognosis of such patients with local relapse is generally dismal due to lack of not safe and effective salvage treatment option for such patients. Another potentially promising approach can be adding boost dose after external beam radiation therapy (EBRT) by brachytherapy for tumors responded poorly against EBRT. Traditionally, intracavitary brachytherapy (ICBT) has been used for local boosting after EBRT for NPC [21,22,23,24,25]. However, because in ICBT, the radioactive source stays in the nasopharyngeal cavity, not directly in the tumor tissue. Therefore, it is usually difficult to deliver adequate dose deeper than $5 \mathrm{~mm}$ beneath the surface of cavity. Recently, a new technique of high-dose-rate interstitial brachytherapy (HDR-ISBT) for NPC as a boost has been reported to escalate local dose, while minimizing unnecessary irradiation to the surrounding normal tissues $[26,27,28]$. In the following, we describe technical aspects of boost HDR-ISBT as dose escalation for NPC patients who respond poorly to the CCRT with EBRT, not salvage HDR-ISBT for tumor persistence or local recurrence.

\section{Technique}

Tumor response to EBRT should be monitored weekly by the usual fiber-optic endoscope. Typically, EBRT is delivered with conventional fractionation of 1.8-2 Gy by
Address for correspondence: Naoya Murakami, MD, PhD, Department of Radiation Oncology, National Cancer Center Hospital, 5-1-1 Tsukiji, Chuo-ku, Tokyo 104-0045, Japan, phone: +81 33542 2511, fax: +81 33545 3567, $₫$ e-mail: namuraka@ncc.go.jp; Guanghui Cheng, MD, PhD, Department of Radiation Oncology, China-Japan Union Hospital of Jilin University, No. 126 Xiantai St., Changchun, China,

๑ e-mail: chengguanghuifl@163.com
Received: 18.02 .2020

Accepted: 10.05 .2020

Published: 30.06 .2020 
intensity-modulated radiation therapy (IMRT) or volumetric modulated arc therapy (VMAT), not to miss the target volume, which correlates to tumor recurrence. When a poor response is suspected, suggesting high probability of residual tumor even after the completion of planned irradiation, magnetic resonance imaging (MRI) should be performed after 50 Gy EBRT or more. If a definite residual tumor is pointed out, boost HDR-ISBT can be attempted after obtaining informed consent from a patient. Although optimal timing of assessing residual disease for boost HDR-ISBT for NPC is not yet decided, guidelines for head and neck cancers stated that in combination of EBRT and ISBT for oropharyngeal cancer, in which ISBT is used as a boost, ISBT is applied 1-2 weeks after EBRT completion and at most within 20 days $[29,30]$. Therefore, it can be said that judging the tumor response to EBRT in the latter part of the treatment and prepare for the ISBT boost is a reasonable approach to perform. Delivering high dose to large volume would directly connect to severe life-threatening late consequences, such as ulceration of the mucosa, abscess formation in the retropharyngeal space, and carotid blowout. The gross tumor volume (GTV) at the time of brachytherapy should be the clinical target volume (CTV) for the HDR-ISBT boost, not the initial GTV before treatment. Since it is generally considered to be dangerous to insert needle applicators into the intracranial residual tumor or skull base, we excluded residual tumors having extensive intracranial and/or skull base invasion. However, if supported by a system, which enabled us to insert applicator needles precisely such as the stereotactic neuro-navigation system, the indication of the HDR-ISBT boost for NPC can be further extended in the future. Organs at risk (OARs) in HDR-ISBT boost for NPC are carotid artery and surface of the nasopharynx. The location and the distance from the surface of nasopharynx to the carotid artery should be checked before needle insertion. Information about anti-coagulant or anti-platelet agents' usage should be taken before the HDR-ISBT boost. The patient's hemoglobin level should be checked beforehand and preparation for swift blood infusion should be performed.

\section{Residual tumor in the lateral part of the nasopharynx}

Most NPC arises from the lateral part of nasopharynx, namely from the Rosenmüller's fossa. As mentioned before, when the residual tumor is in the Rosenmüller's fossa, the organ at risk, to which crucial attention must paid is the carotid artery. Other OARs such as the brainstem, eyes, spinal cord, optic nerves, optic chiasm, etc. are typically further away from the applicators; therefore, brachytherapy dose contribution to these OARs would be minimal because of the inverse-square law. In this technique, CT is used both to determine depth and direction of needle insertion during preplanning, and to guide the actual needle implantation during operation. Before the interstitial needle insertion, the location of carotid artery and the distance from the surface of lateral wall of the nasopharynx and the carotid artery must be checked and measured by image modalities with contrast enhancement agents to avoid the injury of carotid artery. To facilitate the optimal needle insertion from the ipsilateral nasal cavity to the laterally located tumors, needle obturators are angled (Figure 1). Our group uses ProGuide $^{\circledR}$ sharp plastic needles (Nucletron, an Elekta company, Elekta AB, Stockholm, Sweden) for HDR-ISBT, and Cheng et al. in their study used metallic needle (Figure $2 \mathrm{C}, \mathrm{D})$. Here, the angle should be kept under 30 degrees, not to obstruct the radioactive source transmission inside of the needle applicator, and a visual mark on the plastic needle within the length to the carotid artery will help to increase the safety of the procedure. Marking on the needle at a certain length from the needle tip can define the depth of the needle, preventing from not going further, which can be observed on a fibro-optic scope. Local anesthesia to both sides of nasal cavity is performed. Under general anesthesia or sedation, with continuous vital sign monitoring, interstitial needle insertion is performed. The fiber-optic endoscope is inserted to the contralateral side of the nasal cavity and the bent needle(s) is (are) inserted through the ipsilateral nasal cavity under fiberoptic endoscope guidance (Figure 2A, B). The location and depth of the needle(s) is (are) determined by the CT. Cheng et al. utilized a real-time image guidance system (Medtronic Navigation Inc., Louisville, USA) to ensure the safety of interstitial needle insertion [27]. Typically, less than 4 catheters are implanted because of limited space in the nasal cavity and nasopharynx, and because of the limited space, the distance between the catheters is less than $1 \mathrm{~cm}$ and optimally $5 \mathrm{~mm}$ apart. As with interstitial brachytherapy for other anatomical sites, an attempt to insert needles parallelly to achieve a favorable dose distribution should be performed. If needle(s) is (are) to be stayed in place for several days, the gauze packing using biodegradable synthetic polyurethane foams, the Naso-

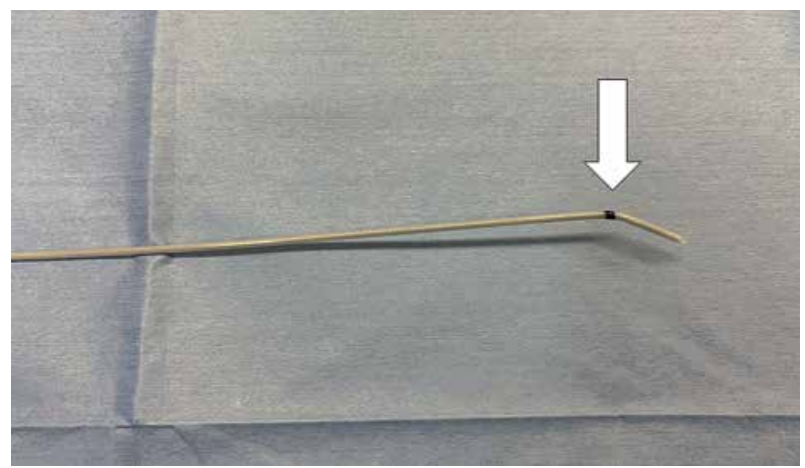

Fig. 1. A 5-French ProGuide ${ }^{\circledR}$ sharp plastic needle (Nucletron, an Elekta company, Elekta AB, Stockholm, Sweden) inferior part, which is slightly bent to facilitate appropriate needle insertion to laterally located tumors. The angle should be kept under 30 degrees, so as not to obstruct the radioactive source transmission inside of the needle applicator. The distance between the ipsilateral internal carotid artery to the surface of the nasopharyngeal mucosa can be measured beforehand on the computed tomography images or magnetic resonance images. A mark on the needle (white arrow) to ensure not to insert the needle deeper will help to insert needles safely 

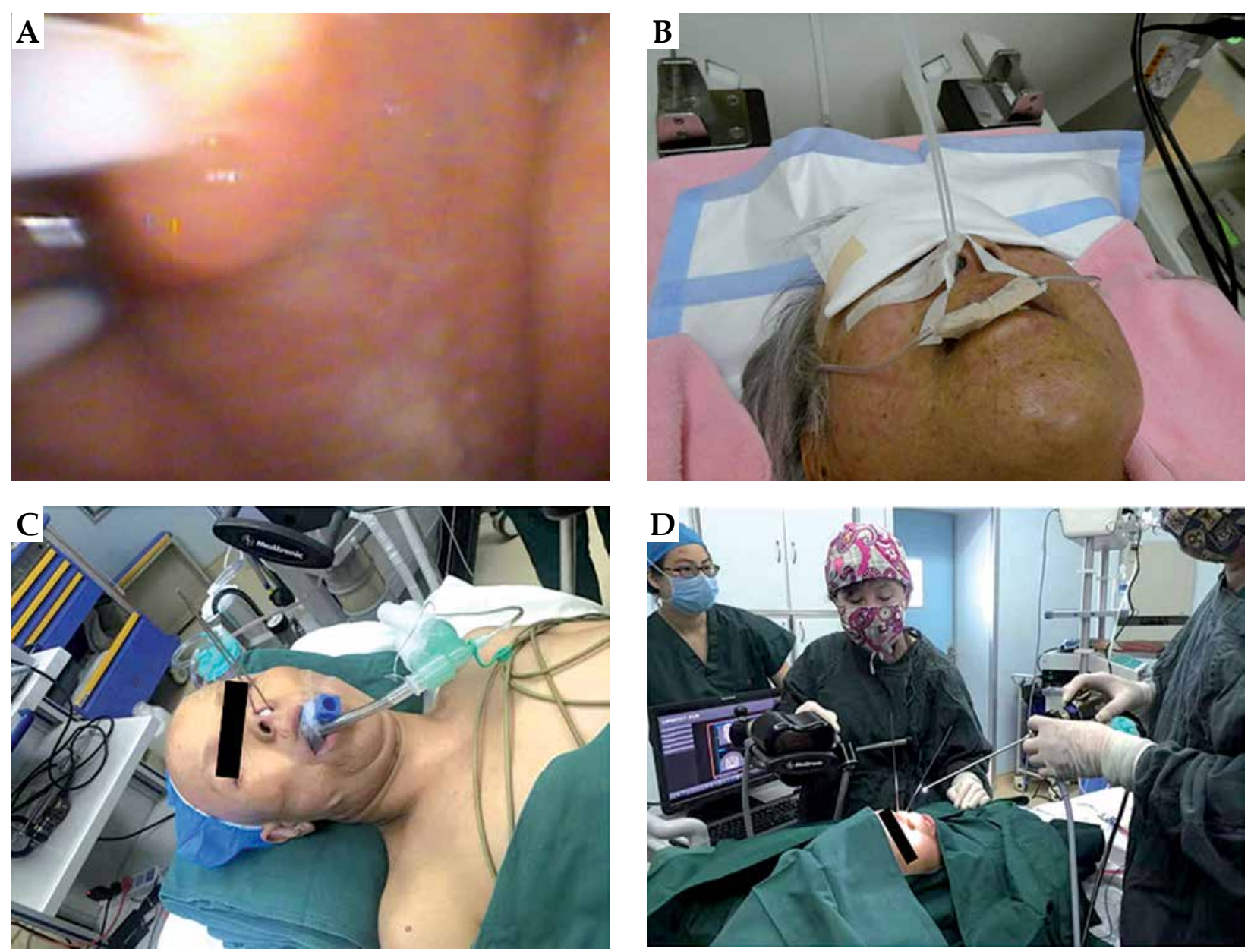

Fig. 2. Under fiber-optic endoscope guidance, the applicator needle is inserted. Once the needle is inserted inside of the nasal cavity, it is difficult to recognize the bent side. Therefore, another mark should be put on the bent side of the handling part of the needle to indicate the bent side. The fiber-optic endoscope is inserted into the contralateral side of the nasal cavity to guide needle applicator insertion. After the tip of the needle is identified by the scope monitor, the needle is rotated laterally and inserted into the lateral nasopharyngeal tissue (A, B). High-dose-rate interstitial brachytherapy (HDR-ISBT) boost for nasopharyngeal cancer (NPC) patient performed in the National Cancer Center Hospital, Tokyo, Japan. One needle in the right lateral side and one in the posterior wall of nasopharynx, respectively, inserted under fiber-optic endoscope guidance (C, D). HDR-ISBT boost for NPC patient performed in the China-Japan Union Hospital of Jilin University, Changchun, China. Two metallic needles were inserted in the left lateral side of nasopharynx

Pore (Polyganics BV, Groningen, The Netherlands), which is used after endoscopic sinus surgery and is packed until the nasopharyngeal cavity, followed by button fixation attached to the needle and sewed to the nasal wing can be used to improve needle fixation during the treatment period. On the other hand, if needle(s) is (are) removed after each brachytherapy session, generally, no fixation is needed because patients are laying down on the treatment table without movement. Certainly, as a measure of precaution, the above-mentioned fixation procedure can be applied for a single irradiation and removal case.

\section{Residual tumor in the ceiling/posterior part of nasopharynx}

When the residual tumor is in the ceiling/posterior part of nasopharynx, needle insertion is available by per-nasal approach with usual straight needles under fiber-optic endoscope guidance. Preparation and the actual procedure are similar to that of previous section, and needle insertion to the midline tumors is much easier than laterally located tumors, because there is no carotid artery or other critical normal structures in the midline.

\section{Residual tumor in the inferior wall of nasopharynx}

In rare cases, NPC can arise from the inferior wall of nasopharynx. When the main tumors are situated in the oral cavity side, they are categorized in soft palate cancer, namely, superior wall of oropharynx. But when the main tumors are in the nasopharyngeal side, they are classified into NPC, arising from the inferior wall of nasopharynx [31]. Similar to soft palate cancer, NPC 
in the inferior wall of nasopharynx can be treated with HDR-ISBT using trans-oral approach. Unless the needle goes to the lateral part of nasopharynx, there is no critical structure to cover the tumor located in the inferior wall of nasopharynx. Under general anesthesia or local anesthesia and sedation, needle insertion is performed. Needle insertion is guided by paranasal endoscope and $\mathrm{CT}$. To assist the airway patency, the nasal airway can be inserted through the nasal cavity (Figure 3). Unlike the Pernot's technique for soft palate cancer, the stability of needle in this per-oral approach is unreliable. Therefore, irradiation was performed only once, and the needles were removed after single treatment. However, because this technique is less invasive than the Pernot's technique performed under local anesthesia, if multiple fractions is needed, multiple implantations can be achieved. Not for nasopharyngeal cancer, but safely performed trans-oral multiple fractions needle insertion for a recurrent oropharyngeal cancer patient with similar approach was previously reported by our group, in which $12 \mathrm{~Gy}$ in 2 fractions of HDR-ISBT was safely applied after 60 Gy of EBRT [32].

\section{Treatment planning}

HDR-ISBT boost for residual NPC is generally performed after delivering 60-70 Gy of EBRT [26,27,28]. Dose calculation should be completed on 3D CT-based image guidance (Figure 4). After needle(s) insertion, CT planning with slice thickness less than $3 \mathrm{~mm}$ is obtained. Our group uses Oncentra Brachy v. 4.5.1 (Nucletron, an ELEKTA company, ELEKTA AB, Stockholm, Sweden) for dose calculation and ${ }^{192}$ Ir remote afterloading system, Microselectron-V2, (Nucletron, an ELEKTA company, ELEKTA AB, Stockholm, Sweden) for dose delivery. Any optimizing method can be used such as geometrical optimization, dose-volume optimization, or inverse planning simulated annealing (IPSA); however, final manual modification checking slice by slice is mandatory. When a cold spot under the prescription dose is found on the CT, additional applicator needle(s) can be inserted to adequately cover the target volume higher than the prescription dose, using previously implanted needle(s) as a landmark. In institutions where MRI is available, MRI fusion is desirable with applicator(s) in place, because soft-tissue resolution of MRI is superior to that of CT. In several guidelines for head and neck brachytherapy, dose per fraction between $2.5 \mathrm{~Gy}$ to $6 \mathrm{~Gy}$ is recommended $[30,33,34,35]$. In the literature concerning HDR-ISBT for NPC, dose per fraction varied from 2 to $7 \mathrm{~Gy}$, and total delivered dose also varied from 11 to $20 \mathrm{~Gy}[26,27,28]$. However, the Chinese group deliver HDR-ISBT boost within 2-3 months after completion of EBRT, more than $10 \mathrm{~Gy}$ was required to control the tumor. Our group deliver HDR-ISBT boost shortly after completion of EBRT, and single fraction of 4 Gy could successfully control a residual tumor that responded poorly to CRT presumably because of shortened total treatment time (a case report currently under revision). Unlike vaginal mucosa, nasopharyngeal mucosa is more likely to develop mucosal ulceration. As mentioned before, the late consequence of such posterior wall mucosa ulceration of nasopharynx could be linked to abscess formation in retropharyngeal space and, in the worst-case scenario, could cause carotid artery blow-out. Therefore, after a full dose of EBRT, the dose per fraction is recommended to be kept under $4 \mathrm{~Gy}$. Additionally, a high dose volume greater than $200 \%$ of the prescribed dose on the surface of mucosa should be securely monitored. If the target volume is large, lowering the dose per fraction should be considered.
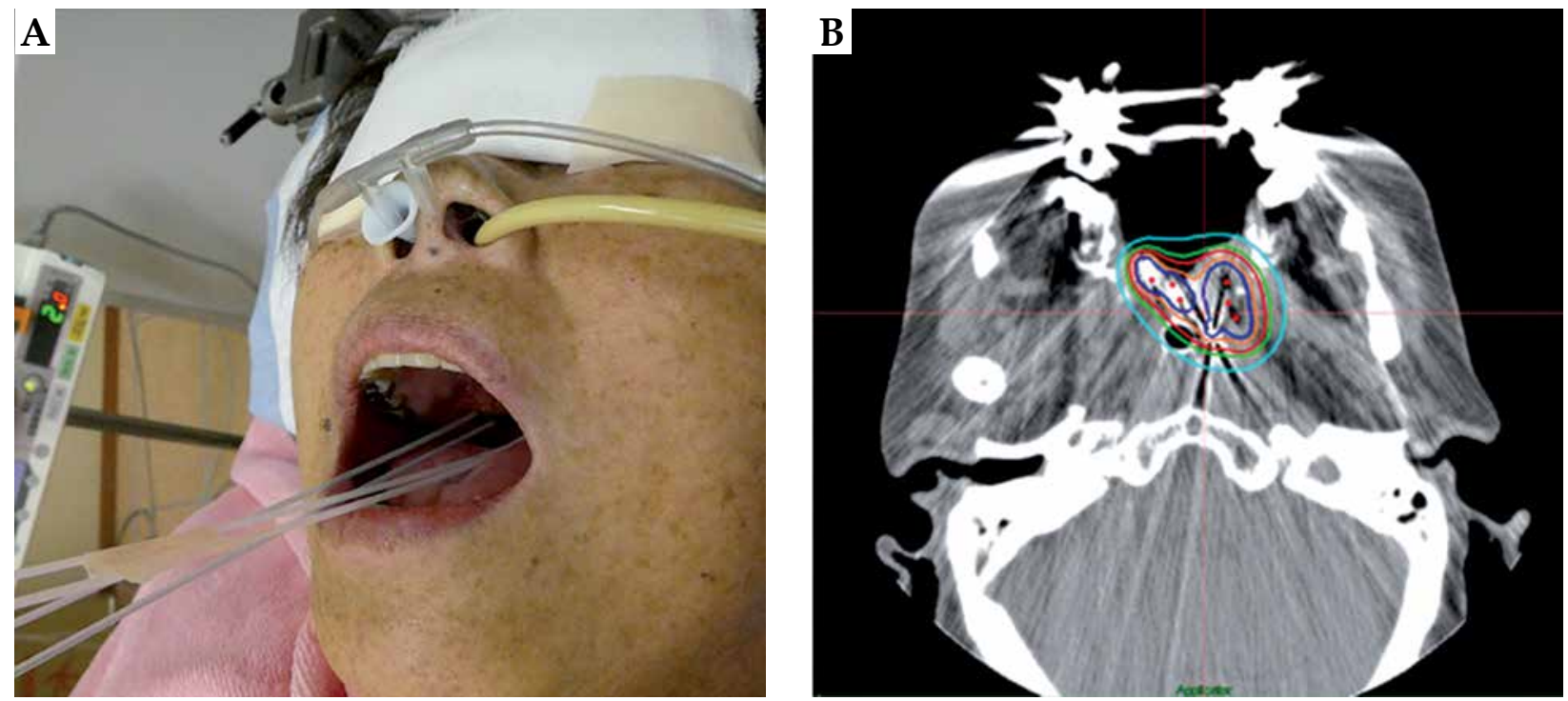

Fig. 3. A) Under local anesthesia and sedation, four 5-French ProGuide ${ }^{\circledR}$ sharp plastic needles (Nucletron, an Elekta company Elekta $\mathrm{AB}$, Stockholm, Sweden) were inserted trans-orally through the tumor located in the inferior wall of nasopharynx. The depth of the needles was determined by CT image; B) Isodose distribution of the interstitial implant with the red and blue line representing the $100 \%$ and $200 \%$ isodose, respectively 


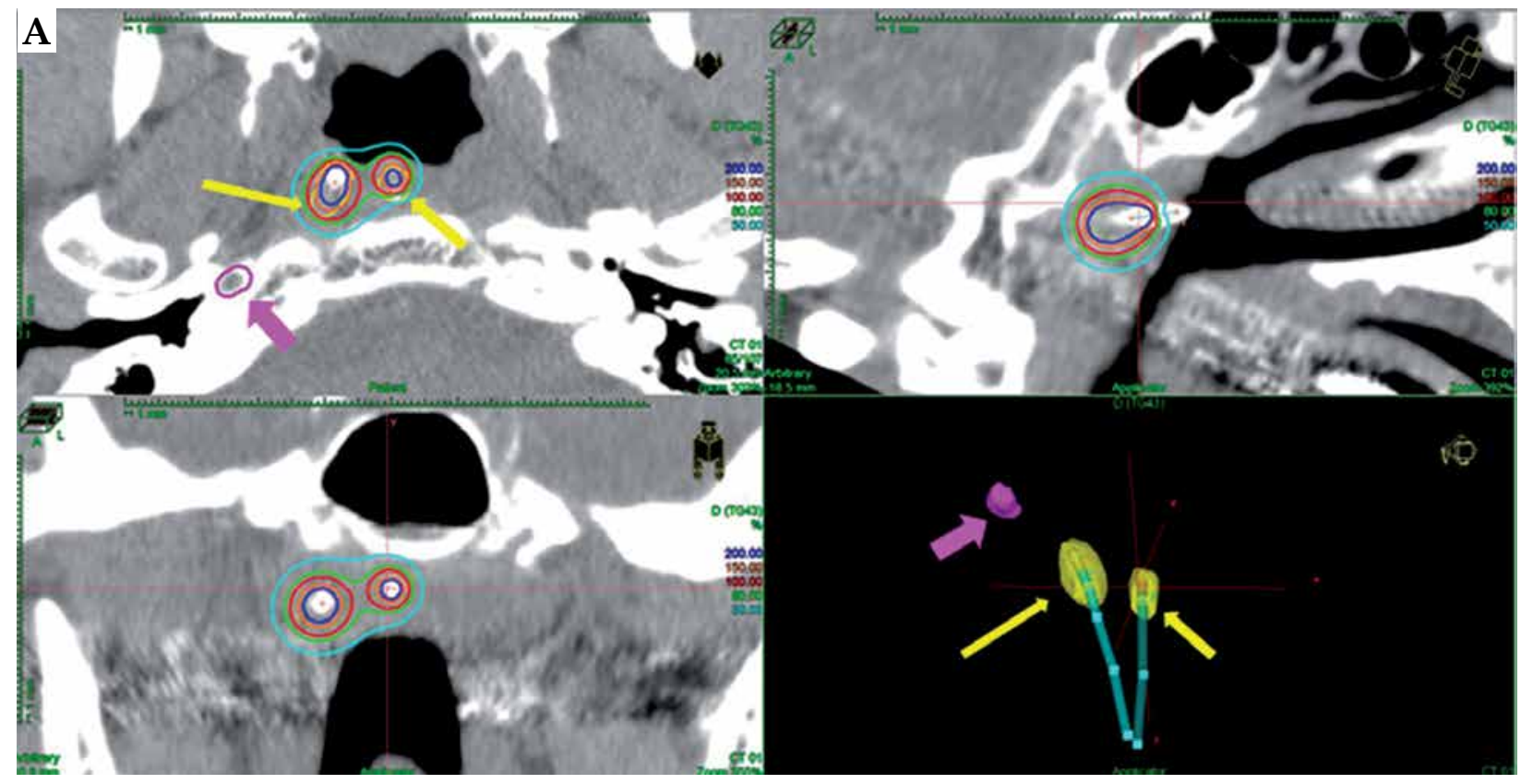

B

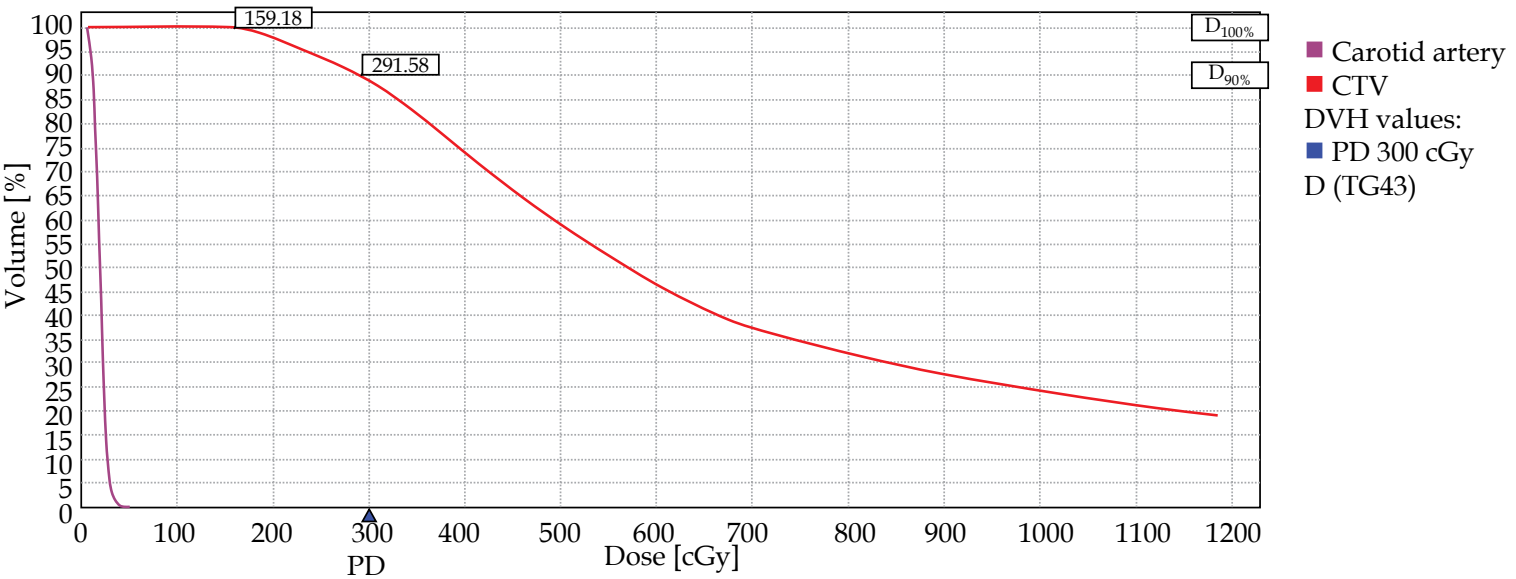

Fig. 4. A) Isodose distribution of the high-dose-rate interstitial brachytherapy (HDR-ISBT) for nasopharyngeal cancer (NPC) patient performed in the National Cancer Center Hospital, Tokyo, Japan. The residual tumor was located on the right side of nasopharynx and the applicator needle was inserted through the right nasal cavity. The red and blue isodose lines represent the $100 \%$ and $200 \%$ isodose, respectively. The pink and yellow arrow correspond to the right carotid artery and the clinical target volume at the time of brachytherapy (CTV-BT), respectively; B) Dose-volume histogram of right carotid artery (pink line) and CTV-BT (red line). Dose calculation was performed using Oncentra Brachy v. 4.5.1 (Nucletron, an ELEKTA company, ELEKTA $\mathrm{AB}$, Stockholm, Sweden). High-dose-rate brachytherapy was performed using ${ }^{192}$ Ir remote afterloading system, Microselectron-V2 (Nucletron, an ELEKTA company, ELEKTA AB, Stockholm, Sweden)

\section{Needle removal}

At the time of needle removal, crucial attention must be paid to bleeding from the puncture site. To facilitate the compression hemostasis, a small portion of gauze soaked with epinephrine can be prepared. When active bleeding is observed after needle removal, prompt compression hemostasis with the epinephrine-soaked gauze is performed under careful continuous vital sign monitoring.

\section{Discussion}

Standard therapy for locally advanced NPC is CCRT, followed by adjuvant chemotherapy or induction chemo- therapy, followed by CCRT [36]. NPC responds well to the chemoradiation therapy and local residual/persistent or local recurrences are uncommon. There are, however, some patients who respond poorly to standard chemoradiation therapy, and it has been reported that about $7.5-14 \%$ of locally advanced NPC patients develop persistent or local relapse after definitive chemoradiation therapy $[18,19,20]$. When local control cannot be achieved, the prognosis of such patients is quite poor, because of limited options to salvage such diseases. Several attempts have been made to salvage local recurrent disease with various modalities; outcomes of salvage surgical resection were reported, but this approach resulted 

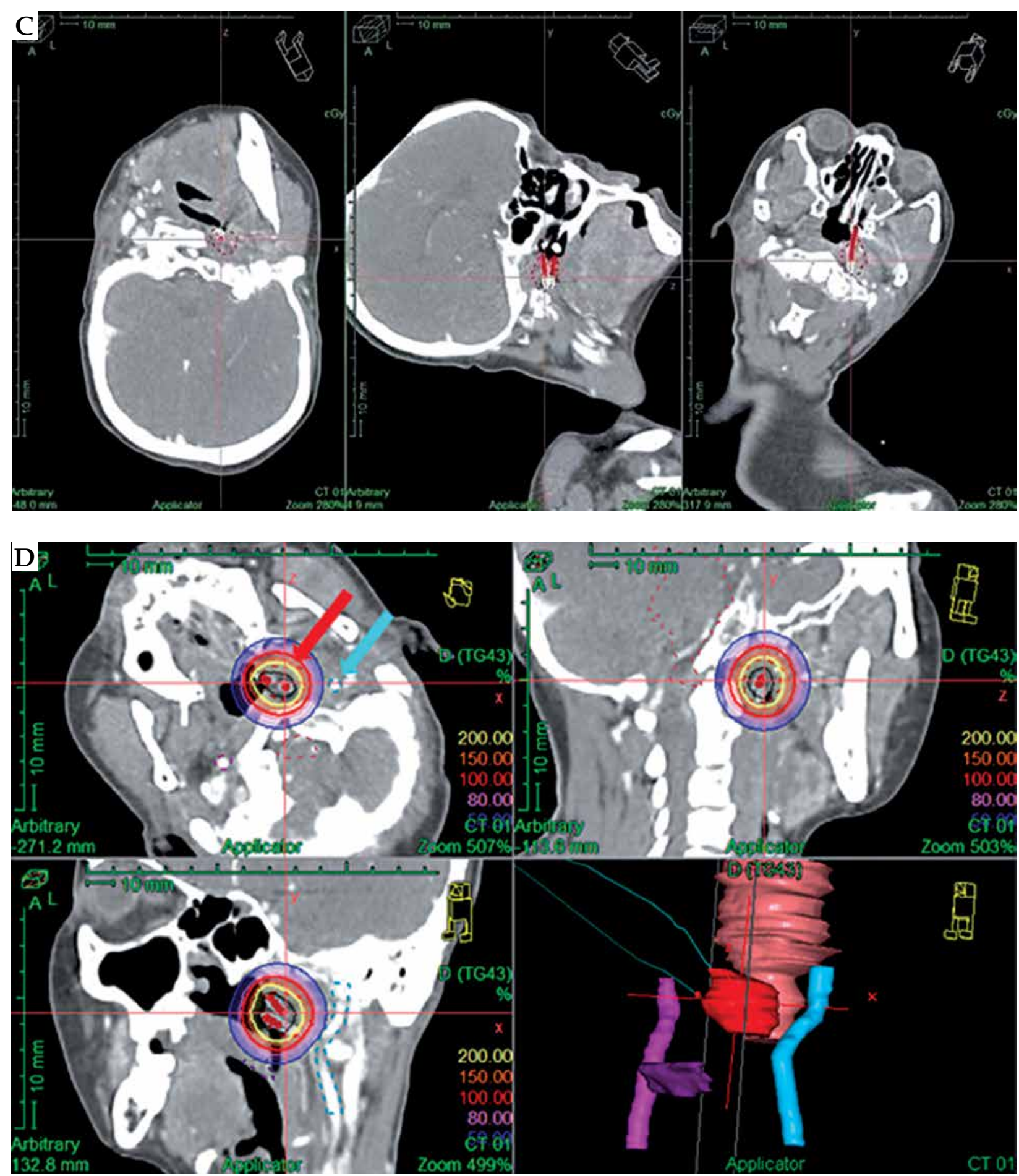

Fig. 4. Cont. C) Axial, sagittal, and coronal images with interstitial needles implanted on the left side of nasopharynx; D) Isodose distribution of HDR-ISBT boost for NPC patient performed in the China-Japan Union Hospital of Jilin University, Changchun, China. Blue and red arrow represent the left carotid artery and the gross tumor volume (GTV), respectively. The blue, purple, red, and yellow isodose lines correspond to $50 \%, 80 \%, 100 \%$, and 200\% isodose, respectively; E) Dose-volume histogram of organs at risk, GTV, and clinical target volume (CTV). GTV, CTV, and carotid arteries are shown in red, green, and blue lines, respectively 


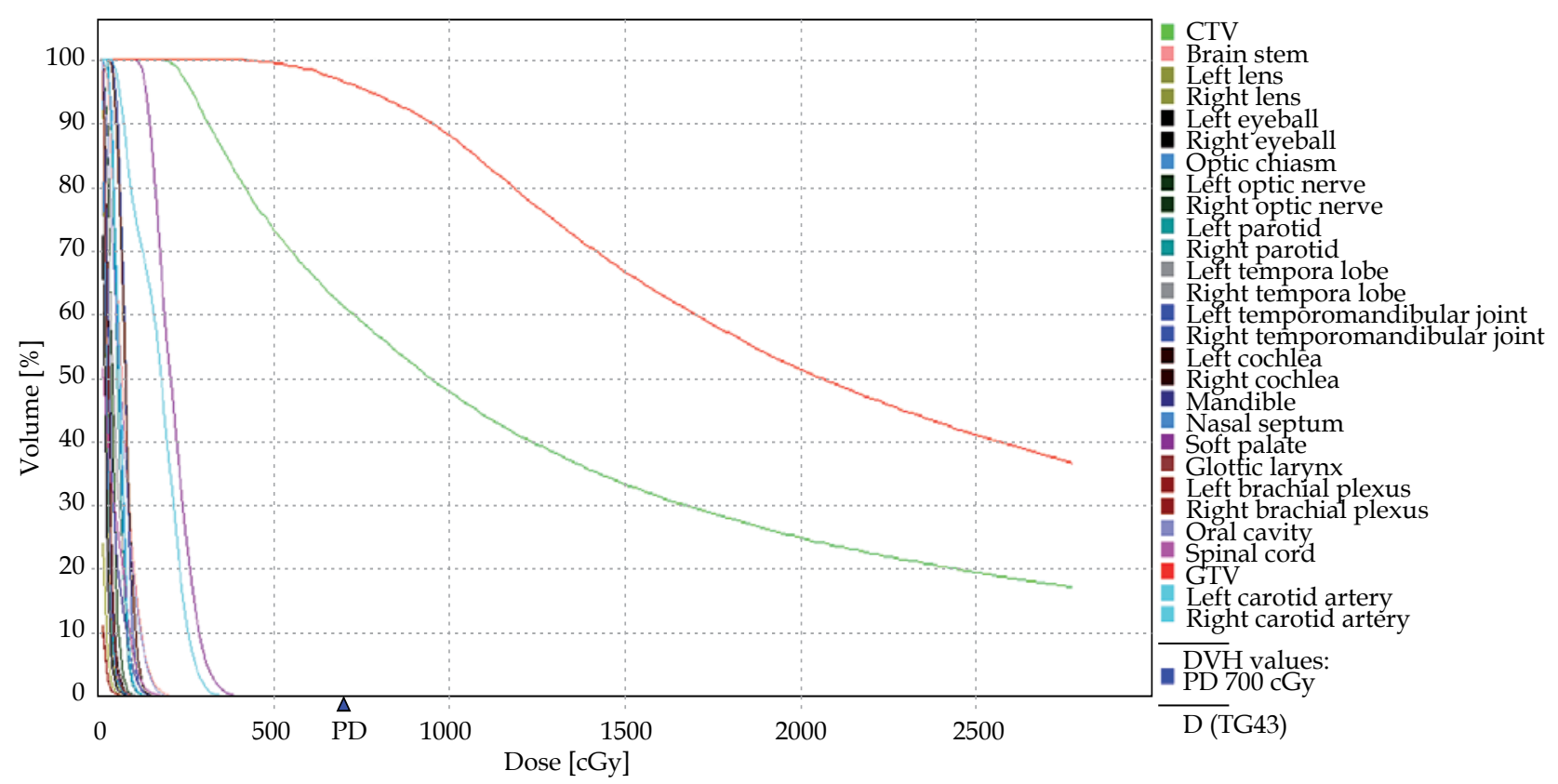

Fig. 4. Cont. E) Dose-volume histogram of organs at risk, GTV, and clinical target volume (CTV). GTV, CTV, and carotid arteries are shown in red, green, and blue lines, respectively

in high complication rates with high positive resection margin (29.0\%) and 5-year disease-free survival was $42 \%$ [37]. Re-irradiation with EBRT has been explored in the form of conventional fractionation using IMRT [38,39] or stereotactic body ablative radiotherapy using the $\mathrm{Cy}$ berKnife (Accuray, Inc., Sunnyvale, CA, USA) [40], but such treatments were associated with severe late toxicities due to accumulated radiation. It was reported by Han et al. that along with recurrent $\mathrm{T}$ stage, tumor volume larger than $38 \mathrm{~cm}^{3}$ and fractional dose larger than 2.3 Gy were associated with late severe toxicities after re-irradiation with IMRT for locally recurrent NPC patients [38]. In a systematic review of literature involving 1,554 patients with re-irradiation for recurrent head and neck cancer, rate of carotid blow-out was reported to be $2.6 \%$, and $76 \%$ of these carotid blow-outs were fatal [41]. Therefore, achieving local control in the initial treatment is of paramount importance in the management of NPC.

Intracavitary brachytherapy (ICBT) has been used for local boosting after external beam radiation therapy (EBRT) for NPC [21,22,23,24,25]. Because in ICBT, the radioactive source stays in the nasopharyngeal cavity and not directly placed inside of the tumor tissue, it is usually difficult to deliver adequate dose deeper than $5 \mathrm{~mm}$ beneath the surface of the nasopharyngeal cavity without delivering dangerously enormous dose to the mucosal surface. If the thickness of the residual tumor is less than $5 \mathrm{~mm}$, the ICBT boost for NPC is an adequate boost modality. However, not all the residual tumors fall into less than $5 \mathrm{~mm}$ thickness. Accordingly, a phase III prospective clinical trial by the International Atomic Energy Agency (IAEA) to determine whether ICBT boost after EBRT improves clinical outcomes in NPC patients has failed to show its efficacy, supposedly because of inadequate irradiation dose coverage of the lateral tumor extension with ICBT [22]. Cumulative knowledge of interstitial brachytherapy
(ISBT) in other anatomical sites [42,43,44,45,46,47] proved that if the thickness of the tumor is larger than $5 \mathrm{~mm}$, it is unreachable with conventional ICBT, and if interstitial needle application is possible, a boost with HDR-ISBT would be a better option to escalate local dose, while minimizing unnecessary irradiation to surrounding normal tissues $[26,27,28]$. A group from the Thomas Jefferson University reported the usefulness of salvage surgery combined with re-irradiation with intraoperative ${ }^{131} \mathrm{Cs}$ permanent brachytherapy implant in recurrent head and neck cancer $[48,49]$. Although, the number of patients investigated in the study was as small as eleven and because the dose fall-off gradient adjacent to the brachytherapy source was extremely steep, only limited volume of carotid artery (less than $1 \mathrm{~mm}^{3}$ ) received $19 \pm 10 \mathrm{~Gy}$, which was acceptable [48], suggesting that brachytherapy is a suitable modality to deliver high dose close to critical structures. Because of a paucity of reports on HDR-ISBT boost for NPC, only limited clinical outcomes were reported so far. However, a group from the Sun Yat-sen University compared clinical results between EBRT alone $(n=101)$ and combination of EBRT and HDR-ISBT boost $(n=40)$ for T2b NPC patients, and they reported better 5-year local failure-free survival in favor of combination group ( $80.2 \%$ vs. $97.5 \%$; $p=0.012)$. Optimal timing of delivering HDR-ISBT is not yet determined. While Chinese groups deliver HDR-ISBT boost within 2-3 months after completion of EBRT, our group tries to perform it shortly after EBRT to reduce the total treatment time, which may facilitate to control the tumor with a smaller additional dose. Obviously, because HDR-ISBT boost to NPC is a relatively new technique, patients should be followed up as long as possible to report late complications related to this treatment. Incidentally, ISBT for NPC itself is not a new technique. In 1984, Vikram et al. reported trans-nasal permanent ${ }^{125}$ I interstitial implantation for NPC patients [50]. They employed a similar technique, however, 
while we inserted needles under trans-nasal endoscopic guidance, they inserted ${ }^{125}$ I seeds trans-nasally under transoral endoscopic guidance, presumably because only the rigid scope was available at that time.

Because neither ultrasonography nor the conventional X-ray fluoroscopy can visualize the relationship between the needle tip and the carotid artery, and once the carotid artery is injured, devastating consequences would follow. Therefore, it is highly emphasized that needle insertion should be performed under CT guidance.

There are so many things needed to be explored with adding HDR-ISBT boost in patients with NPC. Appropriate timing for judging the response of EBRT and HDR-ISBT boost application, appropriate fractionation, correct total dose, applicable target volume definition, and dose constraints for surrounding normal OARs are unknown. In the section of technique, guidelines about the combination of EBRT and HDR-ISBT for oropharyngeal cancer were cited $[29,30]$. Certainly, there is a difference between the combination of EBRT and HDR-ISBT for oropharyngeal cancer and NPC, which is presented in this article. In oropharyngeal cancer, HDR-ISBT boost is used to complement lower EBRT dose and HDR-ISBT is intended to be applied from the starting point, while in NPC, the decision of adding HDR-ISBT boost is based on the tumor response. In the authors' opinion, a dose of 70 Gy was just determined historically by surrounding normal structures' tolerance dose for EBRT, not by the tumor response itself. Therefore, while most NPC respond well to conventional 70 Gy of CCRT, some tumors respond poorly to 70 Gy of EBRT, and such tumors would need more than $70 \mathrm{~Gy}$ to be controlled. It was demonstrated that in different anatomic sites such as prostate cancer or uterine cervical cancer, brachytherapy boost can safely deliver a much higher dose than EBRT due to its specific feature of steep dose fall-off. Regarding the response of NPC, if the weekly response by the fiberscope can be carefully monitored, it is possible to distinguish between good and poor responders as well as to recognize intermediate responders. For intermediate responders, it is recommended to wait for the final response 8-12 weeks after the completion of $70 \mathrm{~Gy}$, because there are patients who respond slowly. HDR-ISBT boost should initially be applied to poor responding patients. However, after an accumulation of knowledge, it could be possible that intermediate responders should also receive HDR-ISBT boost to obtain favorable local control. But until substantial knowledge is accumulated, the boost should be spared. Finally, its efficacy should be validated in a future prospective clinical trial with NPC patients who respond poorly to the conventional CCRT with or without HDR-ISBT boost application. Since in South China and Southeast Asian countries, many NPC patients can be found, it is highly expected that further important studies on HDR-ISBT boost for NPC will be performed by researchers (brachytherapists) from those countries.

\section{Acknowledgments}

This study was partially supported by the Japan Agency for Medical Research and Development (AMED, 19ck0106305h0003), the National Cancer Center Research and Development Fund (26-A-18 and 26-A-28), and the Project of Social Development Division in Department of Science and Technology of Jilin Province (20190303151SF).

\section{Disclosure}

Dr. Jun Itami receives personal fees from HekaBio and from kay J outside the submitted work.

The rest of the authors report no conflict of interest.

\section{References}

1. Network NCC. NCCN Guidelines Version 1.2020 Head and Neck Cancers. 2020.

2. Bansal A, Ghoshal S, Oinam AS et al. High-dose-rate interstitial brachytherapy in early stage oral tongue cancer - 15 year experience from a tertiary care institute. J Contemp Brachytherapy 2016; 8: 56-65.

3. Inoue T, Inoue T, Teshima T et al. Phase III trial of high and low doserateinterstitialradiotherapyforearlyoral tonguecancer.Int J Radiat Oncol Biol Phys 1996; 36: 1201-1204.

4. Shibuya H, Hoshina M, Takeda M et al. Brachytherapy for stage I \& II oral tongue cancer: an analysis of past cases focusing on control and complications. Int J Radiat Oncol Biol Phys 1993; 26: 51-58.

5. Takacsi-Nagy Z, Oberna F, Koltai P et al. Long-term outcomes with high-dose-rate brachytherapy for the management of base of tongue cancer. Brachytherapy 2013; 12: 535-541.

6. Yoshida K, Takenaka T, Akiyama H et al. Three-dimensional image-based high-dose-rate interstitial brachytherapy for mobile tongue cancer. I Radiat Res 2014; 55: 154-161.

7. Akiyama H, Major T, Polgar C, Takacsi-Nagy Z. Dose-volume analysis of target volume and critical structures in computed tomography image-based multicatheter high-doserate interstitial brachytherapy for head and neck cancer. J Contemp Brachytherapy 2017; 9: 553-560.

8. Yoshida K, Yamazaki H, Kotsuma T et al. Edema worsens target coverage in high-dose-rate interstitial brachytherapy of mobile tongue cancer: a report of two cases. J Contemp Brachytherapy 2017; 9: 66-70.

9. Murakami N, Ueno T, Yatsuoka W et al. Dose coverage comparison between "interstitial catheter-only" and "hybrid intracavitary-interstitial brachytherapy" for early stage squamous cell carcinoma of the buccal mucosa. I Contemp Brachytherapy 2018; 10: 486-491.

10. Strnad V. Treatment of oral cavity and oropharyngeal cancer. Indications, technical aspects, and results of interstitial brachytherapy. Strahlenther Onkol 2004; 180: 710-717.

11. Yamazaki H, Yoshida K, Yoshioka Y et al. High dose rate brachytherapy for oral cancer. J Radiat Res 2013; 54: 1-17.

12. Yoshimura R, Shibuya H, Miura M et al. Quality of life of oral cancer patients after low-dose-rate interstitial brachytherapy. Int J Radiat Oncol Biol Phys 2009; 73: 772-778.

13. Pernot M, Hoffstetter S, Peiffert D et al. Role of interstitial brachytherapy in oral and oropharyngeal carcinoma: reflection of a series of 1344 patients treated at the time of initial presentation. Otolaryngol Head Neck Surg 1996; 115: 519-526.

14. Al-Mamgani A, Levendag PC, van Rooij P et al. Intensity-modulated radiotherapy followed by a brachytherapy boost for oropharyngeal cancer. Head Neck 2013; 35: 1689-1697.

15. Bussu F, Tagliaferri L, Mattiucci G et al. Comparison of interstitial brachytherapy and surgery as primary treatments for nasal vestibule carcinomas. Laryngoscope 2016; 126: 367-371. 
16. Chan KCA, Woo JKS, King A et al. Analysis of Plasma Epstein-Barr Virus DNA to Screen for Nasopharyngeal Cancer. N Engl J Med 2017; 377: 513-522.

17. Kim KY, Le QT, Yom SS et al. Clinical utility of Epstein-Barr virus DNA testing in the treatment of nasopharyngeal carcinoma patients. Int J Radiat Oncol Biol Phys 2017; 98: 996-1001.

18. Sun Y, Li WF, Chen NY et al. Induction chemotherapy plus concurrent chemoradiotherapy versus concurrent chemoradiotherapy alone in locoregionally advanced nasopharyngeal carcinoma: a phase 3, multicentre, randomised controlled trial. Lancet Oncol 2016; 17: 1509-1520.

19. Zhang Y, Chen L, Hu GQ et al. Gemcitabine and cisplatin induction chemotherapy in nasopharyngeal carcinoma. $N$ Engl J Med 2019; 381: 1124-1135.

20. Murakami N, Mori T, Kubo Y et al. Prognostic impact of immunohistopathologic features in definitive radiation therapy for nasopharyngeal cancer patients. J Radiat Res 2020; 61: 161168.

21. Leung TW, Tung SY, Wong VY et al. Nasopharyngeal intracavitary brachytherapy: the controversy of $\mathrm{T} 2 \mathrm{~b}$ disease. Cancer 2005; 104: 1648-1655.

22. Rosenblatt E, Abdel-Wahab M, El-Gantiry $M$ et al. Brachytherapy boost in loco-regionally advanced nasopharyngeal carcinoma: a prospective randomized trial of the International Atomic Energy Agency. Radiat Oncol 2014; 9: 67.

23. Wu J, Guo Q, Lu JJ et al. Addition of intracavitary brachytherapy to external beam radiation therapy for T1-T2 nasopharyngeal carcinoma. Brachytherapy 2013; 12: 479-486.

24. Levendag PC, Keskin-Cambay F, de Pan C et al. Local control in advanced cancer of the nasopharynx: is a boost dose by endocavitary brachytherapy of prognostic significance? Brachytherapy 2013; 12: 84-89.

25. Wang CC. Improved local control of nasopharyngeal carcinoma after intracavitary brachytherapy boost. Am J Clin Oncol 1991; 14: 5-8.

26. Wan XB, Jiang R, Xie FY et al. Endoscope-guided interstitial intensity-modulated brachytherapy and intracavitary brachytherapy as boost radiation for primary early $\mathrm{T}$ stage nasopharyngeal carcinoma. PLoS One 2015; 10: e90048.

27. Zhang $X, W u ~ N$, Zhao Z et al. Fibre-optic endoscope-guided three-dimensional high-dose-rate interstitial brachytherapy for residual nasopharyngeal carcinoma after conventional external beam radiotherapy. J Contemp Brachytherapy 2019; 11: 243-249.

28. Ren YF, Gao YH, Cao XP et al. 3D-CT implanted interstitial brachytherapy for $\mathrm{T} 2 \mathrm{~b}$ nasopharyngeal carcinoma. Radiat Oncol 2010; 5: 113

29. Takacsi-Nagy Z, Martinez-Mongue R, Mazeron JJ et al. American Brachytherapy Society Task Group Report: Combined external beam irradiation and interstitial brachytherapy for base of tongue tumors and other head and neck sites in the era of new technologies. Brachytherapy 2017; 16: 44-58.

30. Kovacs G, Martinez-Monge R, Budrukkar A et al. GEC-ESTRO ACROP recommendations for head \& neck brachytherapy in squamous cell carcinomas: 1st update - Improvement by cross sectional imaging based treatment planning and stepping source technology. Radiother Oncol 2017; 122: 248-254.

31. Sobin LH, Gospodarowicz MK, Wittekind C (eds.). TNM Classification of Malignant Tumours, $7^{\text {th }}$ ed. Wiley-Blackwell 2011.

32. Murakami N, Yoshimoto S, Nakamura S et al. Per-oral interstitial brachytherapy catheter insertion for boost in case of recurrent tonsillar carcinoma: dosimetry and clinical outcome. BJR Case Rep 2020; 6: 20190059.

33. Kovacs G. Modern head and neck brachytherapy: from radium towards intensity modulated interventional brachytherapy. J Contemp Brachytherapy 2015; 6: 404-416.
34. Mazeron JJ, Ardiet JM, Haie-Meder C et al. GEC-ESTRO recommendations for brachytherapy for head and neck squamous cell carcinomas. Radiother Oncol 2009; 91: 150-156.

35. Nag S, Cano ER, Demanes DJ et al. The American Brachytherapy Society recommendations for high-dose-rate brachytherapy for head-and-neck carcinoma. Int J Radiat Oncol Biol Phys 2001; 50: 1190-1198.

36. Network NCC. Head and Neck Cancers (Version 2.2018). In. 2018.

37. King WW, Ku PK, Mok CO, Teo PM. Nasopharyngectomy in the treatment of recurrent nasopharyngeal carcinoma: a twelve-year experience. Head Neck 2000; 22: 215-222.

38. Han F, Zhao C, Huang SM et al. Long-term outcomes and prognostic factors of re-irradiation for locally recurrent nasopharyngeal carcinoma using intensity-modulated radiotherapy. Clin Oncol (R Coll Radiol) 2012; 24: 569-576.

39. Chua DT, Sham JS, Leung LH, Au GK. Re-irradiation of nasopharyngeal carcinoma with intensity-modulated radiotherapy. Radiother Oncol 2005; 77: 290-294.

40. Seo Y, Yoo H, Yoo S et al. Robotic system-based fractionated stereotactic radiotherapy in locally recurrent nasopharyngeal carcinoma. Radiother Oncol 2009; 93: 570-574.

41. McDonald MW, Moore MG, Johnstone PA. Risk of carotid blowout after reirradiation of the head and neck: a systematic review. Int J Radiat Oncol Biol Phys 2012; 82: 1083-1089.

42. Murakami N, Kato T, Miyamoto Y et al. Salvage high-doserate interstitial brachytherapy for pelvic recurrent cervical carcinoma after hysterectomy. Anticancer Res 2016; 36: 24132421.

43. Murakami N, Kobayashi K, Kato T et al. The role of interstitial brachytherapy in the management of primary radiation therapy for uterine cervical cancer. I Contemp Brachytherapy 2016; 8: 391-398.

44. Murakami N, Kobayashi K, Shima S et al. A hybrid technique of intracavitary and interstitial brachytherapy for locally advanced cervical cancer: initial outcomes of a single-institute experience. BMC Cancer 2019; 19: 221.

45. Sekii S, Murakami N, Kato T et al. Outcomes of salvage highdose-rate brachytherapy with or without external beam radiotherapy for isolated vaginal recurrence of endometrial cancer. J Contemp Brachytherapy 2017; 9: 209-215.

46. Umezawa R, Murakami N, Nakamura S et al. Image-guided interstitial high-dose-rate brachytherapy for locally recurrent uterine cervical cancer: A single-institution study. Brachytherapy 2018; 17: 368-376.

47. Yanping B, Murakami N, Shima S et al. Image-guided highdose-rate interstitial brachytherapy for recurrent rectal cancer after salvage surgery: a case report. J Contemp Brachytherapy 2019; 11: 343-348.

48. Walsh A, Hubley E, Doyle L et al. Carotid dosimetry after re-irradiation with (131)Cs permanent implant brachytherapy in recurrent, resected head and neck cancer. J Contemp Brachytherapy 2019; 11: 221-226.

49. Rodin J, Bar-Ad V, Cognetti D et al. A systematic review of treating recurrent head and neck cancer: a reintroduction of brachytherapy with or without surgery. J Contemp Brachytherapy 2018; 10: 454-462.

50. Vikram B, Hilaris B. Transnasal permanent interstitial implantation for carcinoma of the nasopharynx. Int J Radiat Oncol Biol Phys 1984; 10: 153-155. 